\title{
Status and prospects of charged lepton flavor violation searches with the MEG-II experiment
}

\author{
Cecilia Voena *i \\ Istituto Nazionale di Fisica Nucleare Sez. di Roma, P.le Aldo Moro 2, 00185 Roma, Italy \\ E-mail: cecilia.voena@romal.infn.it
}

The MEG experiment took data at the Paul Scherrer Institut (PSI) in the years 2009-2013 and published the most stringent limit on the charged lepton flavor violating decay $\mu \rightarrow e \gamma: \mathrm{BR}(\mu \rightarrow$ $e \gamma)<4.2 \times 10^{-13} @ 90 \%$ C.L.

The MEG detector is currently being upgraded in order to reach a sensitivity of $\sim 4 \times 10^{-14}$, which corresponds to an improvement of one order of magnitude. The basic idea of MEG-II is to achieve the highest possible sensitivity by making the maximum use $\left(7 \times 10^{7}\right.$ muons/s $)$ of the available muon intensity at PSI with an improved detector, keeping the background at a manageable level. The status of the MEG-II detector and the current schedule will be presented. MEG-II, together with the next generation charged lepton flavor violation experiments Mu3e $\left(\mu^{+} \rightarrow e^{+} e^{-} e^{+}\right)$at PSI and Mu2e and COMET ( $\mu \rightarrow e$ conversion) at Fermilab and J-PARC respectively, will reach very high sensitivities in the next years. Accelerator upgrades are expected that will make muon beams with intensities of the order of $10^{10}$ muons/s feasible. At this extremely high beam rates, new detector concepts should be adopted in order to overcome the accidental background. Some future directions will be discussed.

The 19th International Workshop on Neutrinos from Accelerators-NUFACT2017

25-30 September, 2017

Uppsala University, Uppsala, Sweden

* Speaker.

${ }^{\dagger}$ on behalf of the MEG-II collaboration 


\section{Introduction}

The searches for lepton flavor violation in charged lepton decays play a crucial role in the hunt for physics beyond the Standard Model of particle interactions (SM). Historically, the negative results of these searches led to the formulation of the SM, in which lepton flavor conservation is an "accidental" symmetry. The discovery of neutrino oscillations already demonstrated that lepton flavor is not an exact symmetry, although the impact on the charged lepton sector is negligible, predicting, for example for the $\mu \rightarrow e \gamma$ decay, a branching raio (BR) of about $10^{-54}$, far away from the current experimental sensitivity. This symmetry is generally broken in many New Physics (NP) models, sometimes leading to predictions just below the present limits (see [1] for a review) .

Thus, an observation of charged lepton flavor violation (cLFV) would be an unambiguous evidence of NP, while upper limits can be used to constrain NP models.

The present best limit on the $\mu \rightarrow e \gamma$ decay, $\operatorname{BR}(\mu \rightarrow e \gamma)<4.2 \times 10^{-13} @ 90 \%$ C.L [2], has been obtained by the MEG collaboration at the Paul Scherrer Institut (PSI, Switzerland) and the MEG detector is currently being upgraded (MEG-II).

Other cLFV decays involving muons are currently being actively considered, $\mu^{+} \rightarrow e^{+} e^{-} e^{+}$ and $\mu \rightarrow e$ conversion [1] which will not be discussed in this contribution.

In a $\mu \rightarrow e \gamma$ experiment muons are stopped in a target in order to exploit the very clean signature of a decay at rest: an $e^{+}$and a $\gamma$ in coincidence moving collinearly back-to-back with their energies equal to half of the muon mass $\left(m_{\mu} / 2=52.8 \mathrm{MeV}\right)$. There are two main sources of background events. The dominant background is due to an accidental coincidence of a positron from a Michel muon decay $(\mu \rightarrow e v \bar{v})$ with an high energy photon, whose source might be either a radiative muon decay (RMD, $\mu \rightarrow e v \bar{v} \gamma$ ), the annihilation in flight of a positron in a Michel decay or the bremsstrahlung from a positron. The other source of background is the RMD when the positron and the photon are emitted almost back-to-back while the two neutrinos carry off little energy. To separate signal from background events, four discriminating variables are commonly used: the positron and photon energy $\left(E_{e+}\right.$ and $\left.E_{\gamma}\right)$ the relative angle $\left(\Theta_{e+\gamma}\right)$ and the relative time $\left(T_{e+\gamma}\right)$, usually combined in a maximum likelihood analysis to enhance the sensitivity.

It can be demonstrated that the accidental background rate $B_{a c c}$ can be expressed as:

$$
B_{a c c} \propto \Gamma_{\mu}^{2} \cdot \delta E_{e+} \cdot \delta E_{\gamma}^{2} \cdot \delta T_{e^{+} \gamma} \cdot \delta \Theta_{e^{+} \gamma}^{2}
$$

where $\Gamma_{\mu}$ is the beam intensity and the quantities indicated with $\delta$ are the detector resolutions. Since the signal is proportional to $\Gamma_{\mu}$, for a given detector, there is an optimal rate that maximizes the sensitivity and there is no advantage in further increase of $\Gamma_{\mu}$.

\section{The MEG-II project}

The MEG-II [3] experiment is an upgrade of the MEG experiment, approved by the PSI research committee in 2013. The basic idea of MEG-II is to achieve the highest possible sensitivity by making maximum use of the available muon intensity at PSI with the basic principle of the MEG experiment but with improved detectors.

A beam of surface $\mu^{+}$is extracted from the $\pi \mathrm{E} 5$ channel of the PSI high-intensity proton accelerator complex, as in MEG, but with an intensity $\Gamma_{\mu}=7 \times 10^{7}$ muons/s, more than twice of 


\begin{tabular}{lcc}
\hline Parameter & \multicolumn{2}{c}{ Resolution } \\
& MEG & MEG-II \\
\hline$E_{e^{+}}(\mathrm{keV})$ & 380 & 130 \\
$\theta_{e^{+}}(\mathrm{mrad})$ & 9.4 & 5.3 \\
$\phi_{e^{+}}(\mathrm{mrad})$ & 8.7 & 3.7 \\
$z_{e^{+}} / y_{e^{+}}(\mathrm{mm}) \mathrm{core}$ & $2.4 / 1.2$ & $1.6 / 0.7$ \\
$E_{\gamma}(\%)(\mathrm{w}>2 \mathrm{~cm}) /(\mathrm{w}<2 \mathrm{~cm})$ & $2.4 / 1.7$ & $1.1 . / 1.0$ \\
$u_{\gamma}, v_{\gamma}, w_{\gamma}(\mathrm{mm})$ & $5 / 5 / 6$ & $2.6 / 2.2 / 5$ \\
$T_{e^{+}}(\mathrm{ps})$ & 122 & 84 \\
& & \\
\hline Efficiency $(\%)$ & & \\
\hline Trigger & $\sim 99$ & $\sim 99$ \\
$\gamma$ & 63 & 69 \\
$e^{+}$ & 30 & 70
\end{tabular}

Table 1: Comparison of the performances of MEG and MEG-II. $u_{\gamma}, v_{\gamma}, w_{\gamma}$ are the photon conversion point coordinates in a special reference system where $w_{\gamma}$ is the reconstructed depth of interaction.

that used in MEG. After the MEG beam transport system, $\mu^{+}$are stopped in a target, which is thinner than MEG's one to reduce multiple scattering of the emitted positrons.

The positron spectrometer uses the COBRA (COnstant Bending RAdius) solenoid, retained from MEG, that with its gradient field sweeps away low momentum $\mathrm{e}^{+}$. Positron tracks are measured by a newly designed, single-volume, low mass cylindrical drift chamber (DC) able to sustain the required rate. The positron time is measured with improved accuracy by a new pixelated timing counter (TC) based on scintillator tiles read out by silicon multipliers. The new design of the spectrometer increases the signal acceptance by more than factor 2 owing to the reduction of the inactive material between DC and TC. The photon energy, time and decay vertex are measured by the upgraded liquid Xenon calorimeter, where the phototubes on the photon entrance face are substituted with smaller ultraviolet sensitive silicon photomultipliers, thus improving the scintillation light collection uniformity and resolution. A novel device for background suppression is introduced: a radiative decay counter, employs plastic scintillator fibers for timing and lyso crystals for energy measurements to identify low momentum positrons associated to the high energy radiative decay photons. The trigger and the data acquisition systems are also upgraded to meet the stringent requirements of an increased number of channels and to cope with higher rate.

A summary of the comparison of the MEG and MEG-II performances is shown in Tab. 1. A sketch of the detector is shown in Fig. 1. The drift chamber is in its final assembly stage and will be transported at PSI in the Spring 2018, while all the other sub-systems have been successfully tested during the 2017 pre-engineering run. A full engineering run is foreseen in 2019 and the expected sensitivity for a 3 -years physics run is $4 \times 10^{-14}$, one order of magnitude better than MEG.

\section{The next generation of $\mu \rightarrow e \gamma$ searches}

There is currently an intense activity at PSI to design a muon beam line with $\Gamma_{\mu}$ exceeding $10^{9}$ muons/s (HiMB project [4]). Other ideas are present worldwide [5, 6]. 


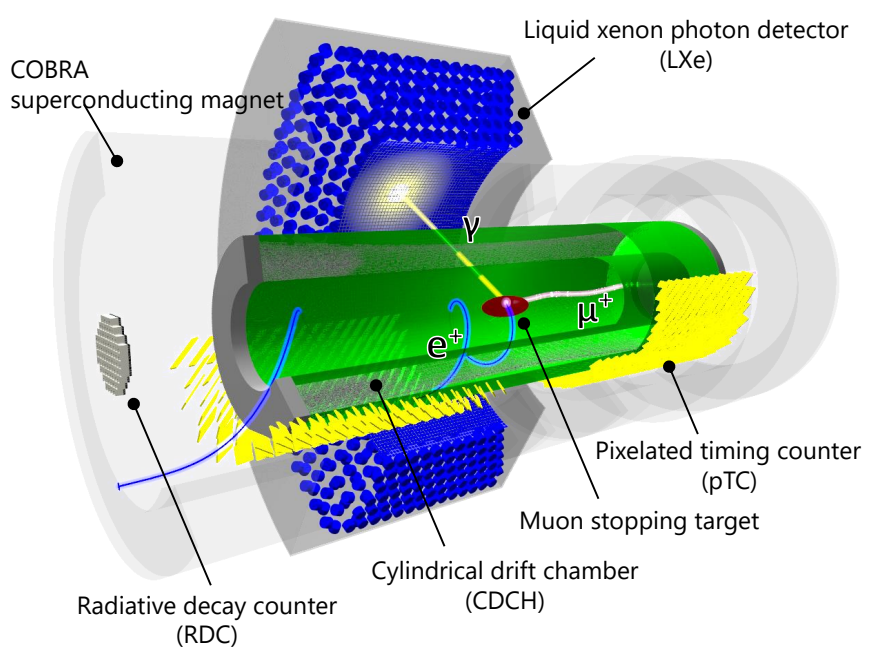

Figure 1: Sketch of the MEG-II detector.

These recent developments will give the possibility to run $\mu \rightarrow e \gamma$ experiments with muon beams one or two orders of magnitude more intense of what presently available.

In order to cope with these very intense muon beams, the detector efficiencies and in particular the resolutions should improve accordingly. A more deepened and quantitative discussion of what presented below can be found in [7].

Concerning the positron, the golden choice is a tracking detector in magnetic field, to measure the positron energy and direction. This approach provides very high detection efficiency; inefficiencies, however, can arise when the track is propagated from the last measurement point to the positron timing detector. The material in front of the positron path ultimately limits the achievable resolutions by multiple scattering and energy loss fluctuations. It is crucial to have the lowest material budget in the target, in any region around the beam-line and in the tracking volume. Gaseous detectors are preferable as extended trackers with respect to silicon detectors, which conversely can be considered to determine precisely the muon decay point and the positron angles. The basic option for the extended tracker is a drift chamber with stereo wires for the longitudinal position measurement as in MEG-II. The drawback of such a detector is possible aging due to the high track rate in the inner layers; this could make, at very high beam rates, a TPC the only choice for a gaseous extended tracker.

Conversely, for photon detection the interplay between efficiency and resolutions has to be carefully considered. In most of the past experiments, a calorimetric technique was adopted, including MEG with its liquid Xenon detector. This approach provides large efficiency, only limited by the amount of material in front of the detector. High light yield and fast response are required to guarantee high energy and time resolutions.

However, there is also the possibility to convert the photon in thin layers $(\sim 0.1$ radiation 
lengths) of high $\mathrm{Z}$ material and then track the $e^{+} e^{-}$pair in a magnetic field. The conversion efficiency is very low (few \%) but this technique provides a very precise energy measurement, an extremely precise conversion point measurement and some information about the direction of the photon. Depending on the sensitivity regimes, these very good resolutions can compensate the loss in efficiency. The photon time can be obtained by measuring the time either of the electron or of the positron with dedicated timing detectors. If one conversion layer is foreseen, one can place thick scintillators at some distance from the converter. In order to stack multiple layers, on the other hand, a layer of active material just behind the converter should provide the required time resolution. Since a thick layer of plastic scintillator would deteriorate unacceptably the photon energy resolution and thin scintillating fibers cannot provide adequate timing resolution, a technological breakthrough is needed here. A possibile solution is given by a new generation of silicon detectors [7].

An estimate of the sensitivity reach has been made using Monte Carlo simulations [7] assuming as benchmark performances those summarized in Tab. 2 .

\begin{tabular}{lcc}
\hline Observable & one $\boldsymbol{\gamma}$ conv. layer & calorimeter \\
\hline$T_{e^{+}}[\mathrm{ps}]$ & 60 & 50 \\
$E_{e}^{+}[\mathrm{keV}]$ & 100 & 100 \\
$E_{\gamma}[\mathrm{keV}]$ & 320 & 850 \\
Efficiency [\%] & 1.2 & 42 \\
\hline
\end{tabular}

Table 2: Expected performances (efficiency and resolutions) for a basic design with different options as discussed in the text.

The results obtained are reported in Fig. 3 where the expected 90\% C. L. upper limit on the $\mu \rightarrow e \gamma$ branching ratio for a 3 -years run is shown as a function of the beam rate. The possibility of having 10 conversion layers and a TPC vertex detector are also considered. The current MEG limit and the expected MEG-II sensitivity are shown as a reference. It can be seen that a $\sim 2 \cdot 10^{-15}$ limit seems to be within reach with $10^{9}$ muons/s while a further increase of the beam rate up to $10^{10}$ muons/s would improve the sensitivity by a factor $\sim 2$.

\section{Summary}

The observation of the $\mu \rightarrow e \gamma$ decay would be an unambiguous sign of New Physics. The MEG experiment set the most stringent limit in the world: $\operatorname{BR}(\mu \rightarrow e \gamma)<4.2 \times 10^{-13} @ 90 \%$ C.L. and the MEG detector is currently being upgraded. The resolutions on the relevant physical variables are expected to improve by about a factor 2 . Those improvements, together with an improvement by a factor 2 in the muon decay rate and in the signal acceptance will bring down the sensitivity by an order of magnitude in 3 years of data taking. A pre-engineering run has been carried out at PSI in Autumn 2017, while a full engineering run with the whole detector is foreseen in 2018 .

Efforts are ongoing at PSI (Switzerland) and elsewhere to develop muon beam-lines with target beam intensities of $10^{9}-10^{10}$ muons/s. The detector requirements to exploit such beam rates are discussed. Sensitivity studies show that in a 3-years run a sensitivity of few $10^{-15}$ is 


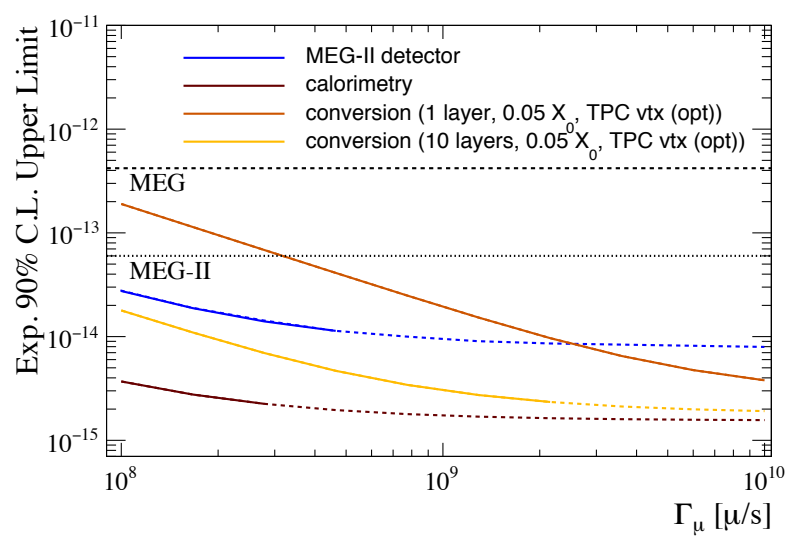

Figure 2: Expected 90\% C. L. upper limit on the $\mu \rightarrow e \gamma$ branching ratio in different scenarios for a 3-years run. Calorimetry and photon-conversion technique are compared. The lines turn from continuous to dashed when the number of background events exceeds 10. The horizontal dashed and dotted lines show the current MEG limit and the expected MEG-II sensitivity. See text for more details.

within reach with poor perspectives of going below $10^{-15}$ even with $10^{10} \mathrm{muon} / \mathrm{s}$. A further step in beam intensity would require a radically new experimental concept.

\section{References}

[1] L. Calibbi, G. Signorelli, Charged lepton flavor violation: an experimental and theoretical introduction, arXiv:1709.00294.

[2] A. M. Baldini et al., Search for the lepton flavor violating decay $\mu \rightarrow e \gamma$ with the full dataset of the MEG experiment, Eur. Phys. J. C76 no. 8, 434 (2016).

[3] A. M. Baldini et al., MEG upgrade proposal, arXiv:1301. 7225.

[4] P. R. Kettle, contribution to Future Muon Sources 2015, University of Huddersfield, United Kingdom.

[5] S. Cook et al., Delivering the word's most intense muon beam, Phys. Rev. Accel. Beams 20 no 3, 030101 (2017).

[6] V. Lebedev et al., The PIP-II reference design report, [PIP-II Collaboration], [FERMILAB-DESIGN-2015-01].

[7] G. Cavoto, et al., The quest for $\mu \rightarrow e \gamma$ and its experimental limiting factors at future high intensity muon beams, arXiv:1707.01805, accepted by Eur. Phys. J. C. 